\title{
Perfil clinico de pacientes internados em unidades de neurologia
}

\section{Clinical profile of patients hospitalized in neurology units}

\author{
Francisco Mayron Morais Soares' • Carlos Lucas Damasceno Pequeno² • Mariana Pereira Maia $^{2}$ \\ Monaliza Ribeiro Mariano ${ }^{3} \bullet$ Rita Neuma Dantas Cavalcante de Abreu ${ }^{4}$ \\ Silvestre Pericles Cavalcante Sampaio Filho ${ }^{5} \bullet$ Italo Rigoberto Cavalcante Andrade $^{6} \bullet$ Julyana Gomes Freitas $^{7}$
}

\begin{abstract}
RESUMO
Objetiva-se identificar o perfil clínico e sociodemográfico de pacientes internados em unidade de neurologia. Trata-se de um Estudo documental, retrospectivo, com abordagem quantitativa. A pesquisa foi realizada em um Hospital Terciário, localizado em Fortaleza, entre o período de dezembro de 2017 e março de 20I8, tendo uma amostra de 184 prontuários. Os dados foram coletados por meio um instrumento com perfil sociodemográfico e clínico e analisados por meio de um Software estatístico. Houve predominância do sexo masculino representando 83,2\%, com idade média entre 38,3, de cor parda $(77,7 \%)$ e natural do interior $(60,3 \%)$ do Ceará. Os diagnósticos médicos mais encontrados foram o de traumatismo cranioencefálico $(54,8 \%$ ) e trauma raquimedular (26\%). Quanto ao desfecho dos pacientes, destaca-se que $88 \%$ obtiveram alta hospitalar, sendo que $28,8 \%$ apresentaram complicações neurológicas. Com uma média de 26 dias de internação hospitalar. Conclui-se o destaque da importância das tecnologias para adquirir todas as informações sobre o paciente e, assim, a partir das suas individualidades, realizar as intervenções necessárias para o cuidado do mesmo.

Palavras-chave: Cuidados de Enfermagem; Enfermagem em Neurociência; Enfermagem de Cuidados Críticos.
\end{abstract}

\begin{abstract}
The objective was to identify the clinical and sociodemographic profile of patients hospitalized in a neurology unit. This is a retrospective, documentary study with a quantitative approach. The study was conducted in a Tertiary Hospital, located in Fortaleza, between December 2017 and March 2018, with a sample of 184 medical records. Data were collected through an instrument with sociodemographic and clinical profile and analyzed using statistical software. There was a predominance of males, representing $83.2 \%$, with a mean age of 38.3 , brown $(77.7 \%)$ and from the countryside $(60.3 \%)$ in Ceará. The most frequent medical diagnoses were traumatic brain injury (54.8\%) and spinal cord trauma (26\%). Regarding the patients' outcome, $88 \%$ were discharged from hospital, and $28.8 \%$ presented neurological complications. With an average of 26 days of hospitalization. The conclusion of the importance of the technologies to acquire all the information about the patient and, from their individualities, to carry out the necessary interventions for the care of the same.
\end{abstract}

Keywords: Nursing Care; Nursing in Neuroscience; Critical Care Nursing.

'Enfermeiro. Mestrando em Enfermagem pela Universidade da Integração Internacional da Lusofonia Afro-Brasileira - UNILAB. Professor Orientador do Laboratório de Tecnologia em Enfermagem. mayronenfo@gmail.com.

²Enfermeiros pela Universidade de Fortaleza.Membro do Laboratório de Tecnologia em Enfermagem.carloslucasdamasceno@hotmail.com e marianamaia_06@ hotmail.com.

${ }^{3}$ Enfermeira. Doutora em Enfermagem pela Universidade Federal do Ceará. Docente da graduação em enfermagem da Universidade da Integração Internacional da Lusofonia Afro-Brasileira - UNILAB. monalizamariano@unilab.edu.br.

${ }^{4}$ Enfermeira. Doutora. Docente da Graduação e do Mestrado Profissional em Enfermagem da Universidade de Fortaleza.rita_neuma@yahoo.com.br.

${ }^{5}$ Enfermeiro. Mestrando em Enfermagem pela Universidade de Fortaleza. silvestre.pericles@yahoo.com.br.

${ }^{6}$ Enfermeiro. Mestre em Saúde Coletiva. Docente da Graduação em Enfermagem da Universidade de Fortaleza. italo_rigoberto@yahoo.com.br.

${ }^{7}$ Enfermeira. Doutora em Enfermagem. Docente da Graduação e do Mestrado Profissional em Enfermagem da Universidade de Fortaleza. Julyana.freitas@unifor.br Autor correspondente: Francisco Mayron Morais Soares. Rua Raimundo de Castro, 775, Centro. 62500-000. Itapipoca/Ceará. (88) 9 965I6I6. mayronenfo@ gmail.com 


\section{INTRODUÇÃO}

As doenças neurológicas são aquelas que acometem o cérebro, medula, nervos e músculos. Estes problemas são multifatoriais e comprometem de forma multissistêmica o corpo humano, tendo como maior frequência o Traumatismo Crânio Encefálicos (TCE) e AcidenteVascular Cerebral (AVC), que se apresentam como a principal causa de morte em jovens adultos no mundo e terceira causa de morte países desenvolvidos, respectivamente. ${ }^{(1-3)}$

O paciente acometido por uma patologia neurológica exige identificação rápida e acompanhamento efetivo da equipe de saúde, a fim de evitar sequelas que se constituem na perda do controle voluntário dos movimentos musculares, problemas sensoriais, incontinência, distúrbios na comunicação e na fala, que prejudica a saúde emocional, física e social do acometido. Tais dificuldades e/ou incapacidades ocorrem em decorrência das diversas doenças, traumas, que podem acometer o sistema nervoso da vítima. ${ }^{(4)}$

Dentro deste contexto, o enfermeiro deve conhecer as alterações que podem ocorrer no doente para poder atuar corretamente, pois grande parte da assistência de enfermagem baseia-se em cuidados, raciocínio clínico e avaliação contínua. A avaliação de enfermagem da condição neurológica do paciente incorpora o monitoramento do nível de consciência, escala de glasgow a reação pupilar, as funções motoras e a observação dos sinais vitais. ${ }^{(4-6)}$

Com isso, compreender as causas e as consequências de uma doença é primordial durante processo de saúde-doença, a fim de estabelecer um diagnóstico e contribuir para a ação de medidas de prevenção, controle, assistência e educação. ${ }^{(7)}$

Nesse contexto, destaca-se aos profissionais de saúde, sobretudo aos enfermeiros, a importância de qualificar o conhecimento prático e teórico frente aos cuidados com pacientes neurológicos, com o intuito de ofertar assistência de qualidade.Assim, conhecer a incidência dos diagnósticos em pacientes neurológicos é coerente com o perfil de morbidade e mortalidade nacional, além de fornecer subsídios para a atuação do enfermeiro com maior segurança nesse campo, a fim de distinguir e prestar atendimento diante às particularidades de cada paciente.

Diante de tais constatações, surgiu o seguinte questionamento: qual o perfil clínico e sociodemográfico de pacientes neurológicos? A partir do exposto, o estudo objetivou identificar o perfil clínico e sociodemográfico de pacientes internados em unidade de neurologia.

\section{METÓDO}

Estudo documental, retrospectivo, com abordagem quantitativa.

A pesquisa foi realizada em um Hospital Terciário, localizado em Fortaleza, Ceará. Este é considerado o maior centro médico de urgência e emergência, tendo duas unidades de internação exclusivas ao paciente neurológico. ${ }^{(8)}$
A amostra utilizada é do tipo probabilístico e sistemático, sendo calculada com base na fórmula para populações finitas, a qual é indicada para o cálculo da amostra para estudos transversais. ${ }^{(9)}$ Com isso, obteve-se uma população de trezentos e cinquenta e cinco (355) prontuários, chegando a um valor total utilizado para amostra do estudo igual a cento e oitenta e quatro (184) prontuários a serem coletados nas unidades de neurologia.

O período da coleta de dados ocorreu entre os meses de dezembro de 2017 e março de 2018 . A coleta foi realizada por meio de um instrumento construído pelos autores, contemplando variáveis referentes ao perfil sociodemográfico e clínico. O preenchimento do formulário realizou-se através do levantamento das informações contidas nos prontuários utilizados pela própria instituição, a qual é preenchida pelos profissionais envolvidos na assistência ao cliente. Participaram do estudo pacientes que atenderam aos seguintes critérios de inclusão: ter sido internado nas unidades de neurologia do referido hospital, ter tido diagnóstico médico, de acordo com a Classificação Internacional de Doenças (CID-I0), confirmado presente no prontuário, ter tido no mínimo 12 horas de internamento no local do estudo. Foram adotados os seguintes critérios de exclusão: prontuário que impossibilite a coleta de dados por ter sido extraviada, não identificada previamente com o nome do paciente ou que esteja ilegível, entre outros.

Os dados obtidos pelo preenchimento dos formulários foram digitados em um software estatístico. As informações obtidas foram apresentadas em tabelas e analisadas conforme literatura pertinente, tendo como estatística descritiva as medidas de tendência central e como analise inferencial qui quadrado e teste de normalidade de Kolmogorov-Smirnov (K-S), aceitando como valor de $p$ resultado menor que $0,05(p<0,05)$. ${ }^{(9)}$

A pesquisa foi autorizada pelo Comitê de Ética em Pesquisa da Universidade de Fortaleza (UNIFOR), sob o parecer $n^{\circ}$ 2.066.492. Foram respeitados todos os preceitos éticos e legais da Resolução n. ${ }^{\circ} 466 / 12$ do ConseIho Nacional de Saúde - CNS.

\section{RESULTADOS}

Realizou-se análise de 184 prontuários de pacientes internados em unidades de neurologia. A tabela abaixo apresenta o perfil sociodemográfico no qual observouse a predominância do sexo masculino, perfazendo um total de 153 (83,2\%). Em relação à idade, constatou-se prevalência de indivíduos com idade inferior 40 anos $(58,2 \%)$, cuja média resultou em 38,3 anos $( \pm 16,6)$. No que se refere à raça/cor em os pacientes se declaravam, em sua maioria, pardos, resultando assim $77,7 \%$. Ao avaliar a procedência identificou-se que $60,3 \%$ eram naturais do interior do estado do Ceará. 
TABELA 1 - Caracterização do perfil sociodemográfico da amostra. Fortaleza/Ce. Brasil, 2018.

\begin{tabular}{|c|c|c|c|c|c|}
\hline Variáveis & $\mathbf{N}$ & $\%$ & Valor de $p$ & IC $95 \%$ & Md (土DP) \\
\hline \\
\hline$<40$ & 107 & 58,2 & $0,01^{*}$ & $35,9-40,7$ & $38,3( \pm 16,6)$ \\
\hline$\geq 40$ & 77 & 41,8 & $0,00^{* *}$ & & \\
\hline $\begin{array}{l}\text { Sexo } \\
\text { Masculino } \\
\text { Feminino } \\
\text { Raça/Cor }\end{array}$ & $\begin{array}{c}153 \\
31\end{array}$ & $\begin{array}{l}83,2 \\
16,8\end{array}$ & $0,00^{*}$ & & \\
\hline Branca & 17 & 9,2 & $000 *$ & & \\
\hline Parda & 143 & 77,7 & $0,00^{\star}$ & & \\
\hline $\mathrm{NHR}$ & 24 & 13,1 & & & \\
\hline \multicolumn{6}{|l|}{ Procedência } \\
\hline Capital (Fortaleza - CE) & 73 & 39,7 & $0,05^{*}$ & & \\
\hline Interior & 111 & 60,3 & & & \\
\hline \multicolumn{6}{|l|}{ Filhos } \\
\hline 0 & 182 & 98,9 & $0,00^{*}$ & & \\
\hline 1 & 2 & 1,1 & & & \\
\hline Destino & & & $0,00^{*}$ & & \\
\hline Alta Hospitalar & 162 & 88,0 & & & \\
\hline Enfermaria & 8 & 4,3 & & & \\
\hline Óbito & 1 & 0,5 & & & \\
\hline Outra Instituição & 3 & 1,6 & & & \\
\hline $\mathrm{NHR}$ & 10 & 5,6 & & & \\
\hline Total & 184 & 100 & & & \\
\hline
\end{tabular}

Md: média; DP: desvio padrão; NHR: não há registro | ${ }^{*}$ Teste de qui quadrado** Teste de normalidade $K-S$

Fonte: Dados da pesquisa

Quando associado ao desfecho, a procedência foi a única variável que não apresentou significância estatística.

Em relação à tabela 2, quanto ao diagnóstico médico em pacientes neurológicos admitidos nas unidades em estudo, os traumas de cabeça foram a principal representação dos estudos e conforme a Classificação Estatística Internacional de Doenças e Problemas Relacionados à Saúde (CID-I0), o traumatismo cranioencefálico (TCE) ou traumatismo cerebral difuso (S06.2), como mais predominante, resultando em $54,8 \%$, seguido do trauma raquimedular (TRM) ou sequelas de traumatismo da medula espinhal (T9I.3) com 26,0\%.

Em relação ao tempo de internação, apresenta-se que a maioria dos pacientes ficaram internados em um tempo superior a 20 dias.

A seguir na tabela 3 , serão apresentados os dados subdivididos pelos sistemas do corpo humano, seguindo a anatomia cefalopodálica.

Foi evidenciado que $54,9 \%$ apresentaram como classificação da escala de coma de Glasgow como leve, referente a 13 a 15 pontos, mas apresentando média de Glasgow 12,28, com desvio padrão de aproximadamente a 3,9. Contudo, ainda ocorreram negligências por parte dos profissionais de enfermagem que não registraram a mesma $(21,7 \%)$ nos prontuários.

No que diz respeito à avaliação pupilar, outro parâmetro utilizado para avaliação neurológica, o estudo em questão obteve uma amostra de 90 prontuários, cujos enfermeiros realizaram registros quanto às informações da pupila. Destes registros, houve predominância das pupilas isocóricas $(43,5 \% ; n=80)$, condição do tamanho normal entre as pupilas.
Quanto ao sistema urinário, no que diz respeito à sondagem vesical de demora (SVD), este procedimento foi registrado pelos profissionais de enfermagem em mais da metade dos prontuários (51,1\%; $\mathrm{n}=94)$.

Referente à avaliação de presença de lesão por pressão (LPP), o estudo traz um total de apenas 27 prontuários contendo registros de enfermeiros quanto o aparecimento dessas lesões ou não, representando $14,7 \%$ da amostra total de 184. Além desses dados, foi constatado também que dos pacientes que adquiriram LPP, em somente em onze prontuários foi realizada a sua classificação quanto ao estágio da lesão.

\section{DISCUSSÃO}

Em relação à idade, os dados apresentados assemeIham-se a outras publicações de estudos referentes ao perfil sociodemográfico de pacientes neurológicos. Em consonância com os resultados apresentados, pesquisas realizadas em Fortaleza-CE acerca do perfil epidemiológico de pacientes com TCE, e em Ananindeua-PA que avaliaram o perfil clínico-epidemiológico de pacientes atendidos em hospital de referência em trauma, demonstraram que o índice de idade dos pacientes iguais ou inferiores a 40 anos foram de $64 \%$ e $72,8 \%$, respectivamente. ${ }^{(10,11)}$

Os dados evidenciados neste estudo referentes ao gênero sexual com predominância no sexo masculino assemelham-se com outros estudos que mostram uma prevalência respectiva de $82 \%$ por Silva et al. ${ }^{(10)}, 88 \%$ por Viégas et al. ${ }^{(11)}$ e $76,2 \%$ por Lima et al. ${ }^{(12)} \mathrm{Em}$ pesquisa realizada especificamente em setor de neurologia, no ano de 2014 , constatou-se que houve também o domínio da classe do sexo masculino em suas coletas, resultando em $58,57 \%$. ${ }^{(13)}$ 
TABELA 2 - Caracterização dos Diagnósticos, sintomas tempo de internação e destino. Fortaleza/Ce. Brasil, 2018.

\begin{tabular}{|c|c|c|c|c|}
\hline Variáveis & $\mathbf{n}$ & $\%$ & Valor de $p$ & Md ( $\pm D P)$ \\
\hline \multicolumn{5}{|l|}{ Diagnóstico Médico } \\
\hline Traumatismo Cranioencefálico & 101 & 54,8 & & \\
\hline Traumatismo Raquimedular & 48 & 26,0 & & \\
\hline Outros & 35 & 19,2 & & \\
\hline \multicolumn{5}{|l|}{ Sinais e Sintomas } \\
\hline Hemorragia & 7 & 3,8 & & \\
\hline Cefaleia & 13 & 7,1 & & \\
\hline Otorréia & 9 & 4,9 & & \\
\hline Fraturas & 29 & 15,8 & & \\
\hline Convulsão & 14 & 7,6 & & \\
\hline Outras & 61 & 33,2 & & \\
\hline NHR & 51 & 27,6 & & \\
\hline \multicolumn{5}{|l|}{ Dias de Internação Hospitalar } \\
\hline$<20$ & 90 & 48,9 & $0,00^{*}$ & $25,4( \pm 19,4)$ \\
\hline$>20$ & 94 & 51,1 & & \\
\hline \multicolumn{5}{|c|}{ Complicações Decorrentes do Trauma } \\
\hline Neurológicas & 53 & 28,8 & & \\
\hline Infecções & 23 & 12,5 & & \\
\hline Outras & 15 & 8,2 & & \\
\hline NHR & 93 & 50,5 & & \\
\hline Total & 184 & 100 & & \\
\hline
\end{tabular}

Fonte: Dados da pesquisa

TABELA 3 - Caracterização do perfil clínico. Fortaleza/Ce. Brasil, 2018.

\begin{tabular}{|c|c|c|c|c|}
\hline Variáveis & $\mathbf{N}$ & $\%$ & Valor de $p$ & Md ( $\pm \mathrm{DP})$ \\
\hline \multicolumn{5}{|l|}{ SISTEMA NEUROLÓGICO } \\
\hline \multicolumn{5}{|l|}{ Escala de Coma de Glasgow } \\
\hline Moderada & 19 & 10,3 & $0,00^{*}$ & $12,2( \pm 3,9)$ \\
\hline Grave & 25 & 13,6 & & \\
\hline \multicolumn{5}{|l|}{ Avaliação Pupilar } \\
\hline Reagentes à luz & 69 & 37,5 & & \\
\hline Não reagentes à luz & 6 & 3,3 & & \\
\hline NHR & 109 & 59,2 & & \\
\hline \multicolumn{5}{|l|}{ Simetria das Pupilas } \\
\hline Isocórica & 80 & 43,5 & & \\
\hline Anisocórica & 10 & 5,4 & & \\
\hline NHR & 94 & 51,1 & & \\
\hline \multicolumn{5}{|l|}{ Escala de Ramsay } \\
\hline 5 & 9 & 4,9 & & \\
\hline 6 & 24 & 13,0 & & \\
\hline \multicolumn{5}{|l|}{ SISTEMA CARDIOVASCULAR } \\
\hline \multicolumn{5}{|l|}{ Frequência Cardíaca } \\
\hline Normocárdico & 105 & 59,2 & $0,02^{*}$ & $85,1( \pm 19,7)$ \\
\hline Taquicárdico & 23 & 12,5 & & \\
\hline \multicolumn{5}{|l|}{ Pressão Arterial } \\
\hline PA Normal & 90 & 48,9 & & \\
\hline PA Elevada & 37 & 20,1 & & \\
\hline Hipertensão Arterial Sistêmica 2 & 40 & 21,7 & & \\
\hline NHR & 17 & 9,3 & & \\
\hline \multicolumn{5}{|l|}{ SISTEMA RESPIRATÓRIO } \\
\hline \multicolumn{5}{|l|}{ Frequência Respiratória } \\
\hline Eupneico & 120 & 65,4 & & \\
\hline Taquipneico & 17 & 9,1 & & \\
\hline \multicolumn{5}{|l|}{ Oxigenoterapia } \\
\hline Cateter Nasal & 3 & 1,6 & & \\
\hline Máscara de Venturi & 13 & 7,1 & & \\
\hline Máscara de $\mathrm{O}_{2}$ com reservatório & 1 & 0,5 & & \\
\hline Nenhum $\quad 2$ & 102 & 55,4 & & \\
\hline NHR & 65 & 35,4 & & \\
\hline \multicolumn{5}{|l|}{ Via Aérea Artificial } \\
\hline Traqueostomizado & 3 & 1,6 & & \\
\hline
\end{tabular}


Tubo orotraqueal

Ventilação Mecânica

Sim

Não

SISTEMA GASTROINTESTINAL

Sonda

Sonda Nasogástrica

Sonda Orogástrica

Sonda Nasoenteral

Nenhuma

NHR

SISTEMA URINÁRIO

Dispositivo Urinário

Espontânea

Sonda Vesical de Alívio

Sonda Vesical de Demora

NHR

SISTEMA TEGUMENTAR

Escala de Braden

Risco Baixo

Risco Moderado

Risco Alto

Lesão Por Pressão (LPP)

Sim

Não

NHR

Total

*Teste de qui quadrado

Fonte: Dados da pesquisa

Salienta-se que o hospital em estudo é referência em atendimentos a pacientes vítimas de traumas no estado do Ceará. É visto que as principais admissões em unidades que atendam a pacientes vítimas de trauma que necessitem de atendimentos neurológicos, são em sua maioria do sexo masculino e de faixa etária jovem adulta, condizendo um fator de risco para acidentes do tipo automobilístico, agressão física (violência doméstica, perfuração por arma de fogo e/ou branca), consumo excessivo de álcool e outras drogas. ${ }^{(14,15)}$

Dados referentes ao estado civil, nível de escolaridade, situação ocupacional e renda mensal não se apresentavam registrados nos prontuários do respectivo Hospital, fato esse que em consonância com inúmeros dados que não apresentam registros, subnotificam possíveis alterações, tornando-o muitas vezes até difícil à associação dos mesmos.

É importante salientar que as notificações referentes às informações dos pacientes, mesmo algumas sendo dados secundários para o tratamento, facilitarão ao reconhecimento do mesmo pela equipe profissional, auxiliando, por fim, cuidados perante as particularidades de cada um. Além de trazer respaldo e resultados positivos para melhora do quadro clínico do paciente. ${ }^{(16)}$

A escala de coma de Glasgow, em inglês Glasgow Coma Scale (GCS), é um dos parâmetros utilizados para realizar a avaliação neurológica dos pacientes, no momento da sua admissão e durante todo o período de internação hospitalar. ${ }^{(10,17)}$

100

Logo, foi evidenciado que $54,9 \%$ apresentaram GCS classificado em leve, referente a 13-15 pontos, mas apresentando média de Glasgow 12,28, com desvio padrão de aproximadamente a 3,9. Contudo, ainda ocorreram negligências por parte dos profissionais de enfermagem que não registraram o GCS (2I,7\%) nos prontuários. Constatou-se ao estudo de Gomes et al. ${ }^{(17)}$ que $58,1 \%$ das fichas de atendimento de enfermagem de 1.960 ocorrências assistidas pelo Serviço de Atendimento Móvel de Urgência do Rio Grande do Norte (SAMU $192 \mathrm{RN}$ ), representavam GCS leve, além do mais, o mesmo autor, informou que em $39 \%$ dessas fichas o profissional de enfermagem não realizou registro dos dados.

No que diz respeito à avaliação pupilar, outro parâmetro utilizado para avaliação neurológica, o estudo em questão obteve uma amostra de noventa (90) prontuários, cujos enfermeiros realizaram registros quanto às informações da pupila. Destes registros, houve predominância das pupilas isocóricas $(43,5 \% ; n=80)$, condição do tamanho normal entre as pupilas. Ratifica-se a esse resultado um estudo realizado a partir de uma amostra com II 3 pacientes, 87 (77\%) apresentaram pupilas isocóricas, enquanto apenas $16(14,2 \%)$ tiveram as pupilas anisocóricas. ${ }^{(18)}$

Em relação à utilização de via aérea artificial e a escala de sedação de Ramsay, a partir do exposto por Barboza, Silva ${ }^{(19)}$, que avaliou o perfil de pacientes internados em unidades de neurologia, os índices apresentados no estudo foram corroborativos, 
entretanto, salienta-se a necessidade de estudos que abordem essas características, pois a literatura encontra-se escassa.

Quanto ao sistema urinário, no que diz respeito sobre a sondagem vesical de demora (SVD), este procedimento foi registrado pelos profissionais de enfermagem em mais da metade dos prontuários ( $51,1 \% ; n=94)$, evidenciando a predominância também do uso desse dispositivo em outras publicações de estudos. Assim, constatou através de Watanabe et al. ${ }^{(20)}$ e Monagran et al. ${ }^{(21)}$ que a relação de pacientes com cateterização vesical de demora resultou em $50,2 \%$ e $47,0 \%$, respectivamente.

Sobre o sistema tegumentar, observamos um baixo índice de registros por parte da equipe de enfermagem nas unidades de neurologia do referido hospital, sobretudo quanto à utilização da Escala de Braden (EB). Essa escala é um parâmetro utilizado para avaliar o risco de o paciente desenvolver lesão por pressão (LPP), auxiliando o enfermeiro quando em processo de decisão de medidas preventivas a serem feitas de acordo com a necessidade e o risco de cada paciente. A escala contempla seis parêmetros: percepção sensorial, humidade, mobilidade e atividade, nutrição, fricção e cisalhamento; onde se pode classificar o paciente com risco ausente, risco baixo, risco moderado, risco alto, ou risco muito alto para desenvolver algum tipo de LPP enquanto período de internamento. ${ }^{(22-24)}$

\section{CONCLUSÃO}

Frente aos achados do estudo, no perfil sociodemográfico e clínico, observou-se que os tipos de diagnósticos que mais prevalecem aos pacientes neurológicos foi o Traumatismo Cranioencefálico (TCE), sendo o mais prevalente na amostra, com predominância do sexo masculino, com média de idade igual a 38,3 anos, oriundos do interior do Ceará. Observou-se ainda que no desfecho final houve uma prevalência de alta hospitalar ou enfermaria, demonstrando um excelente cuidado prestado a esses pacientes.

Durante a execução desse estudo, foram identificadas algumas limitações que serão expostas a seguir: a ocorrência da ausência de tópicos específicos para os estudos; a subnotificação de várias variáveis; a dificuldade de acesso aos prontuários, que por não serem ainda informatizados, geraram bastante trabalho na coleta.

Nesse contexto, destaca-se a importância das tecnologias para adquirir todas as informações sobre o paciente e, assim, a partir das suas individualidades, realizar as intervenções necessárias para o cuidado do mesmo. Os profissionais enfermeiros destacam-se nessa situação ao poder pôr em prática a sistematização da assistência de enfermagem (SAE), em virtude de sistematizar todas as etapas do cuidado ao paciente, sobre o conhecimento do seu perfil e as intervenções de enfermagem a serem voltadas para o mesmo. Sendo a SAE uma tecnologia fundamental para compilar dados clínicos dos pacientes. 


\section{REFERÊNCIAS}

I. Magalhães ALG, Souza LC, Faleiro RM, Teixeira AL, Miranda AS. Epidemiologia do traumatismo cranioencefálico no Brasil. Revista Brasileira de Neurologia. 2016; 53(2): I5-22.

2. Monteiro LF, Frasson MZ,Wrsesinski A, Bardini AVLS, Lin J, Fernandes AF. Caracterização dos pacientes com traumatismo cranioencefálico grave admitidos em um hospital terciário. Arquivo Catarinense de Medicina. 2016; 45(3): 02-I6.

3. Grumann ARS, Schoeller SD, Martini AC, Forner S, Baroni GC, Horongozo BD. Características das pessoas com acidente vascular encefálico atendidas em um centro de referência estadual. Rev Fund Care Online. 2017; 9(2): 3। 5-320.

4. Oliveira JG, Damasceno KG, Souza LP, Lima MG. Perfil clínico epidemiológico e os principais rótulos diagnósticos de enfermagem aos pacientes internados com acidente vascular cerebral em um hospital de grande porte na região sul da Amazônia legal. Revista Amazônia Science \& Health. 2016; 4(3): 3-II.

5. Santos CW, Vancini-Campanharo CR, Lopes MCBT, Okuno MFP, Batista REA. Avaliação do conhecimento de enfermeiros sobre a escala de coma de Glasgow em um hospital universitário. Einstein.2016; 14(2): 213-218.

6. Nogueira LS, Padilha KG, Silva DV, Lança EFC, Oliveira EM, Sousa RMC. Pattern of nursing interventions performed on trauma victims according to the Nursing Activities Score. Revista Escola Enfermagem USP. 2015; 49(2): 29-35.

7. Sallum AM, Sousa RM. Diagnósticos de enfermagem em vítimas de trauma nas primeiras seis horas após o evento.ACTA Paulista Enfermagem. 2012; 25(2): 256-262.

8. Fortaleza - Ceará. Catálogo de serviços da Prefeitura de Fortaleza. Instituto Dr. José Frota (IJF). 2018.

9. Hulley SB, Cumming SR, Browner WS, Grady DG, Newman TB. Delineando a pesquisa clínica: uma abordagem epidemiológica. 4. ed. Porto Alegre:Artmed, 2015.

10. Silva JAS, Souza ARS, Aline RF, Cavalcante TMC. Traumatismo cranioencefálico no município de Fortaleza. Enfermagem Foco. 2017; 8 (I): 22 - 26.

I I. Viégas MLC, Pereira ELR, Targino AA, Furtado VG, Rodrigues DB. Traumatismo cranioencefálico em um hospital de referência no estado do Pará, Brasil: prevalência das vítimas quanto a gênero, faixa etária, mecanismo de trauma e óbito. Arquivo Brasileiro Neurocirurgia. 2013; 32(I): I5-8.

I2. Freitas, L. S., Lucena, S. K. P., \& Costa, I. K. F. Prevalência de complicações em pessoas com estomias urinárias e intestinais. Revista Enfermagem Atual. 20I7; 82 (20): 55-6I.

13. Batista AJ, Torres JR, Schwind RTF, Peternella FN, Mendes FV. Perfil epidemiológico do setor de neurologia da clínica escola de fisioterapia da Faculdade Ingá no ano de 2013. Revista UNINGÁ. 2014; I7(2): II-I5.

14. Monteiro LF, Frasson MZ,Wrsesinski A, Bardini AVLS, Fernandes AF. Caracterização dos pacientes com traumatismo cranioencefálico grave admitidos em um hospital terciário. Arquivo Catarinense de Medicina. 2016; 45(3): 2-I6.

15. Silva AMA, Shama SFM. Epidemiologia do trauma em atendimentos do SAMU novo Hamburgo/RS no primeiro trimestre de 2015. Revista Saúde e Pesquisa. 2017; 10(3): 539-548.

16. Araújo JS, Nascimento HM, Farre AGMC, Brito RO, Santos JPA, Vasconcelos TTS. Conhecimento dos enfermeiros sobre evento adverso e os desafios para a sua notificação. Cogitare Enfermagem. 20I6; 2 I (4): 0 I-08.

17. Gomes ATL, Silva MF, Dantas BAS, Miranda JMA, Melo GSM, Dantas RAN. Perfil epidemiológico das emergências traumáticas assistidas por um serviço pré-hospitalar móvel de urgência. Enfermería Global. 20 16; I6(I): 384-405.

18. Praça WR, Matos MCB, Magro MCS, Hermann PRS. Perfil epidemiológico e clínico de vítimas de trauma em um hospital do Distrito Federal, Brasil. Revista Prevenção de Infecção e Saúde. 20 I7; 3(I): I-7.

19. Barboza DS, Silva RGM. Sistema de Classificação de pacientes em uma Unidade de Terapia Intensiva Neurológica. Journal Health NPEPS. 20 16; I (2): I 97-207.

20. Watanabe EM,Almeida VFA, Ottunes AF, Dessunti EM, Pieri FM, Martins EAP, Kerbauy G. Impacto das infecções relacionadas à assistência à saúde em pacientes acometidos por trauma. Semina: Ciências Biológicas e da Saúde. 2015; 36(I): 89-98.

2I. Monaghan SF, Heffernan DS, Thakkar RK, Reinert SE, Machan JT, Connolly MD, Gregg SC, Kozloff MS, Adams CA Jr, Cioffi WG. The Development of a Urinary Tract Infection Is Associated With Increased Mortality in Trauma Patients. The Journal Of Trauma: Injury, Infection, and Critical Care. 20I I; 7I (6): I569-I574.

22. Borghard AT, Prado TN, Araújo TM, Rogenski NMB, Bringuente MEO. Evaluation of the pressure ulcers risk scales with critically ill patients: a prospective cohort study. Revista Latino-Americana Enfermagem. 20I5; 23(I): 28-35.

23. Roa DZM, Parra DI, Camargo-Figuera FA.Validación e índices de calidad de las escalas de Braden y Norton. Gerokomos. 2017; 28(4): 200-204.

24. Caldini LN, Silva RA, Melo GAA, Pereira FGF, Frota NM, Caetano AJ. Intervenções e resultados de enfermagem para risco de lesão por pressão em pacientes críticos. Revista da Rede de Enfermagem do Nordeste (Online). 2017; 18(5): 598-605. 